\title{
Changes in long non-coding RNA transcriptomic profiles after ischemia-reperfusion injury in rat spinal cord
}

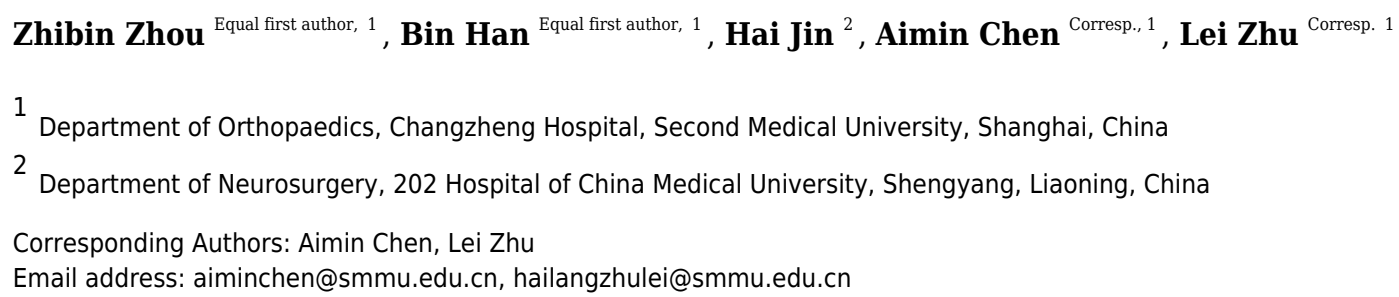

With the aim of exploring expression profiles and biological functions of long non-coding RNA (IncRNA) and mRNAs after ischemia-reperfusion injury (SCII), differentially expressed IncRNAs (DElncRNAs) and mRNAs (DEmRNAs) in rat spinal cords were identified following SCIl through high-throughput RNA sequencing. In total, 1455 IncRNAs and 6707 mRNAs were observed to be differentially expressed (|Fold Change| $\geq 2$ and $\mathrm{P}<0.05$ ) after SCII, including 761 up-regulated and 694 down-regulated IncRNAs and, 3772 up-regulated and 2935 down-regulated mRNAs. Gene ontology and KEGG pathway analysis showed that the DElncRNAs and DEmRNAs were implicated in many different biological processes and pathways. Further, IncRNA-mRNA co-expression networks were built to explore the potential roles of these DEIncRNAs. Our results demonstrate genome-wide IncRNA and mRNA expression patterns in spinal cords after SCII, which may play vital roles in post-SCII pathophysiological processes. These findings are important for future functional research on the IncRNAs involved in SCII and might be critical for providing new insight into identification of potential targets for SCll therapy. 


\section{Changes in long non-coding RNA transcriptomic profiles 2 after ischemia-reperfusion injury in rat spinal cord} 3

4 Zhi-bin Zhou ${ }^{1, \dagger}$, Bin Han ${ }^{1, \uparrow}$, Hai Jin ${ }^{2, \dagger}$, Ai-min Chen ${ }^{1, *}$, Lei Zhu ${ }^{1, *}$

5

61 Department of Orthopaedics, Changzheng Hospital, Second Military Medical University, 7 Shanghai, China

82 Department of Neurosurgery, 202 Hospital of China Medical University, Shenyang, Liaoning, 9 China

$\dagger$ These authors contributed equally to this work.

* Corresponding Author:

Lei Zhu

No. 415, Fengyang road, Huangpu district, Shanghai, 200001, China

Email address: hailangzhulei@smmu.edu.cn

Ai-min Chen

No. 415, Fengyang road, Huangpu district, Shanghai, 200001, China

Email address: aiminchen@smmu.edu.cn 


\section{Abstract}

22 With the aim of exploring expression profiles and biological functions of long non-coding RNA

23 (lncRNA) and mRNAs after ischemia-reperfusion injury (SCII), differentially expressed

24 lncRNAs (DElncRNAs) and mRNAs (DEmRNAs) in rat spinal cords were identified following

25 SCII through high-throughput RNA sequencing. In total, 1455 lncRNAs and 6707 mRNAs were 26 observed to be differentially expressed ( $\mid$ Fold Change $\mid \geq 2$ and $\mathrm{P}<0.05)$ after SCII, including 761

27 up-regulated and 694 down-regulated lncRNAs and, 3772 up-regulated and 2935 down-regulated 28 mRNAs. Gene ontology and KEGG pathway analysis showed that the DElncRNAs and 29 DEmRNAs were implicated in many different biological processes and pathways. Further, 30 lncRNA-mRNA co-expression networks were built to explore the potential roles of these 31 DElncRNAs. Our results demonstrate genome-wide lncRNA and mRNA expression patterns in 32 spinal cords after SCII, which may play vital roles in post-SCII pathophysiological processes.

33 These findings are important for future functional research on the lncRNAs involved in SCII and 34 might be critical for providing new insight into identification of potential targets for SCII 35 therapy. 


\section{Introduction}

38 Spinal cord ischemia-reperfusion injury (SCII) is a serious complication following surgeries that

39

40

41

42

43

44

45

46

47

48

49

50

51

52

53

54

55

56

57

58

59

60

61

62

63

64

65

66

67

68

69

70

71

72

73

74

75

implicates the descending and thoracoabdominal aorta, and can lead to major impairments to functioning of the bowel, bladder, sex and lower extremities(Wan et al., 2001). Although great efforts have been made to develop medical therapeutics for SCII, no efficacious pharmacological or surgical intervention is currently available in clinic to attenuate this injury $(X u$ et al., 2014;

Zhu et al., 2015). Thus, a better mechanistic understanding of SCII is still needed for developing neuroprotective strategies to this devastating complication.

The pathological process of SCII includes two phases: the immediate phase is related to acute ischemia, while the delayed phase involves both ischemic cellular death and reperfusion injury (Zhu et al., 2013). The immediate phase cannot be changed, but injuries caused in the delayed phase can possibly be mitigated by effective therapy. Numerous studies have shown that apoptosis, amino acid toxicity, and calcium overload induced by ischemia-reperfusion injury contribute to neuronal cell death in the pathological processes of SCII(Fan et al., 2011; Ni, Cao \& $\mathrm{Liu}, 2013)$, however, the pathophysiology of SCII varies from time to time. For example, cytokines, which have been proven to play critical role in the delayed phase of SCII, were previously reported to peak in the serum at the early stage of reperfusion (within the first 24 hours), while studies further found that most of thm actually showed a biphasic response (peaked at 6 and 36 to 48 hours)(Smith et al., 2012). Besides that, current evidence also suggests that the spinal cord tissue at the late stage (48 hours) following SCII showed severer and more typical histological feature, which consistent with the degree of functional impairment of the hind limb(Hasturk et al., 2009; Smith et al., 2012). Therefore, revealing the changes in pathophysiology of SCII at late stage of reperfusion in the delayed phase might be of great help in understanding the biological mechanism of SCII.

Long non-coding RNA (lncRNA) represents a class of transcripts longer than 200 nucleotides, but with no coding function(Mercer, Dinger \& Mattick, 2009). Accumulating evidence has shown that lncRNAs have various biological functions in RNA processing, transcription and translation and act as important gene expression regulators(Cech \& Steitz, 2014). Numerous reports have highlighted that aberrant expression of lncRNAs may be crucial in physiological and pathological processes of various human diseases, including myocardial infarction(Wu et al., 2018), osteoarthritis(Liu et al., 2014), diabetic nephropathy(Wang et al., 2018) and cancers(Liu et al., 2018; Wang et al., 2018; Zheng et al., 2018). In recent years, studies on the function of lncRNA in SCII have also gradually increased. Liu et al proved that hydrogen sulfide upregulated lncRNA CasC7 and reduced neuronal cell apoptosis in SCII(Liu et al., 2018), Wang et al revealed that lncRNA MALAT1 played a neuroprotective role in a rat SCII via regulating miR-204(Qiao et al., 2018), and a resesrch group indentified expression profiling of IncRNAs in the spinal cord at the early stage of reperfusion ( $2 \mathrm{~h}$ post-cardiac I/R) by high-throughput RNA sequencing(Wang et al., 2019). However, lncRNA expression pattern and its roles in SCII at the late stage of reperfusion have not been reported yet.

Peer) reviewing PDF | (2019:08:39942:2:1:NEW 20 Nov 2019) 
76 Here, to further investigate the pontial roles of IncRNA in SCII, we screened genome-wide

77 expression patterns of lncRNAs and mRNAs in spinal cords from SCII and control rats via RNA

78 sequencing. Several differentially expressed lncRNAs (DElncRNAs) and mRNAs (DEmRNAs)

79 were validated through quantitative reverse transcription polymerase chain reaction (qRT-PCR).

80 In addition, we performed gene ontology (GO) and KEGG pathway analyses and built co-

81 expression networks to explore the functions of DElncRNAs and DEmRNAs. These results

82 provide a basis for future functional research on the lncRNAs involved in SCII and might be

83 critical for providing new insights into identification of potential targets for SCII therapy.

\section{Materials \& Methods}

85 Spinal cord ischemia reperfusion injury model establishment

86 Male Sprague-Dawley rats (weight, 200-250g) were purchased from the Animal center of the

87 Second Military Medical University (Shanghai, China). All animal experiments were performed

88 according to the guidelines of the Animal Ethics Committee of the Second Military Medical

89 University (Shanghai, China). Thirty rats were randomly divided into the SCII group and sham

90 group (nine in each). In SCII group, the SCII model was established as previously described(Li et

91 al., 2014). After identification of the aortic arch branches, ischemia was achieved by cross-

92 clamping the aortic arch for $14 \mathrm{~min}$. Obstruction was confirmed by a $90 \%$ blood flow decrease in

93 the tail artery using a Laser Doppler Bloodflow Imager (Perimed, Stockholm, Sweden). The

94 clamps were released to reperfuse for $48 \mathrm{~h}$. Rats in sham group that underwent the same

95 procedure without occlusion were used as controls.

96 RNA extraction and high-throughput RNA sequencing

97 Spinal cord segments between L4 and L6 were harvested 48 hours after reperfusion. In total, six

98 samples (three from the SCII group and three from the sham group) were subjected for high-

99 throughput RNA sequencing. For each sample, three random rats spinal cord tissues in the

100 same group were mixed into one sample before RNA extraction. Total RNA was extracted using

101 the mirVana miRNA Isolation Kit (Ambion, Texas, USA) following the manufacturer's protocol.

102 RNA integrity was evaluated using the Agilent 2100 Bioanalyzer (Agilent, California, USA).

103 The samples with RNA Integrity Number (RIN) $\geq 7$ were subjected to the subsequent analysis.

104 The libraries were constructed using TruSeq Stranded Total RNA with Ribo-Zero Gold (Illumina,

105 California, USA) according to the manufacturer's instructions. After validating the size and

106 purity by Agilent Technologies 2100 Bioanalyze (Agilent, California, USA), these libraries were

107 sequenced on the Illumina HiSeqTM 4000 sequencing platform (Illumina, California, USA) and

108150 bp paired-end reads were generated. Libraries construction and RNA-sequencing were

109 performed by OE Biotech (Shanghai, China).

110 Bioinformatics analysis

111 We obtained about 99 million raw RNA-sequencing reads from each sample. First, raw RNA-

112 Seq data were flattened by removing adapter sequences and filtering out low quality reads using

113 Trimmomatic software(Bolger, Lohse \& Usadel, 2014). The clean reads were obtained by quality

114 detection of the obtained reads through fastqc software

115 (www.bioinformatics.babraham.ac.uk/projects/fastqc/), and then they were mapped to the rat 
116 reference genome (Rattus_norvegicus.Rnor_6.0) using hisat2(Kim et al., 2015). The transcript 117 abundances of IncRNA and mRNA were quantified by normalized expression values as FPKM 118 (Fragments Per kb Per Million Reads) using bowtie2(Langmead \& Salzberg, 2012) and 119 eXpress(Roberts \& Pachter, 2013). To identify differentially expressed lncRNAs or mRNAs 120 between the spinal cord after ischemia reperfusion injury and the controls, differential 121 expression analyses were performed using DESeq package. The $p$-value $<0.05$ and |fold change| $122 \geq 2$ were selected as the criteria for significantly DElncRNA or DEmRNA.

\section{Quantitative real-time PCR (qRT-PCR)}

124

125

126

127

128

129

130

131

132

133

134

135

136

137

138

139

140

141

142

143

144

145

146

147

148

149

150

151

152

153

154

155

To validate the reliability of the RNA sequencing results, We randomly selected and analyzed 8 DElncRNAs and 8 DEmRNAs by qRT-PCR analysis. L4-L6 spinal cord tissues were collected from the SCII and sham groups ( $\mathrm{n}=6$ per group). Total RNA was extracted using the TRIzol reagent (Invitrogen, NY, USA) and reversed transcribed into cDNA using random primers. Quantification of lncRNA and mRNA was performed using an ABI PRISM ${ }^{\circledR} 7500$ Sequence Detection System (Applied Biosystems, Forster, CA, USA). Relative lncRNA expression was normalized to the U6 expression and the expression of mRNA level was normalized to GAPDH using the $2-\Delta \Delta \mathrm{CT}$ method. Three independent experiments were conducted for each sample. The primer sequences are shown in Table 1.

\section{Functional and pathway enrichment analyses}

GO analysis and KEGG pathway analyses were conducted to predict the potential functions of DEmRNAs and DElncRNAs based on their co-expressed DEmRNAs. GO analysis was performed to explore potential functions of genes and gene products from three aspects: biological process (BP), cellular component (CC), and molecular function (MF). KEGG pathway analysis was conducted to predict the involvement of the differentially expressed genes in the biological pathways. The top 10 enriched GO terms and top 20 enriched pathways were ranked by enrichment score (-log10 (p-value)) identified by the Database for Annotation, Visualization, and Integrated Discovery (DAVID; https://david.ncifcrf.gov/).

\section{Co-expression of IncRNAs/mRNAs and functional prediction}

The functions of lncRNAs were predicted by annotating the function of the co-expressed mRNAs. A co-expression network was built to explore the interaction between DElncRNAs and DEmRNAs in SCII pathogenesis. The Pearson's correlation coefficient (PCC) was calculated between the expression levels of each DElncRNA-DEmRNA. We then selected co-expression DElncRNA-DEmRNA pairs with value of $|\mathrm{PCC}| \geq 0.80$ and $\mathrm{p}$-value $<0.05$ for network construction(Alkan et al., 2017).

Genomic localizations of the paired lncRNAs and mRNAs were identified for cis prediction. The co-expression nearby gene, which is less than $100 \mathrm{~kb}$ upstream or downstream from the lncRNA, can act as the potential target regulated by the lncRNA in a cis manner, while a trans-regulator is one that does not meet this criterion. The RIsearch-2.0 software(Alkan et al., 2017) was used to identify target genes in trans, with the parameter set as the base number of direct interactions between IncRNA and $m R N A \geq 10$ and free energy $\leq-50$.

\section{Results}


156 High throughput RNA sequencing and genome-wide read mapping

157 L4 to L6 spinal cord segments were harvested for total RNA isolation and Illumina TruSeq 158 library construction (Data available at GEO: GSE138966). 95.49M, 96.94M and 96.77M high 159 quality sequence reads were obtained from three samples of the SCII group, and 95.94M, $16095.22 \mathrm{M}$ and $96.43 \mathrm{M}$ clean reads were generated from three control tissues of the sham-operated

161

162

163

164

165

166

167

168

169

170

171

172

173

174

175

176

177

178

179

180

181

182

183

184

185

186

187

188

189

190

191

192

193

194

195 group (Table 2). All clean reads were mapped to the rat reference genome using hisat2. Nearly $98 \%$ of the reads mapped to the rat genome; $75.25 \%-81.13 \%$ of the reads mapped to unique genomic regions among the aligned fragments (Table 2), indicating the reliability of the data. In total, $25328 \mathrm{lncRNAs}$ and $55760 \mathrm{mRNAs}$ were identified.

\section{The expression profiling of IncRNA and mRNA in the SCII model}

In this study, 1455 lncRNAs and 6707 mRNAs were significantly differentially expressed, with $\mid$ fold change $\mid \geq 2.0, \mathrm{P}<0.05$ and FDR $<0.05$. In total, there were 761 up-regulated lncRNAs, 694 down-regulated lncRNAs, 3772 up-regulated mRNAs, and 2935 down-regulated mRNAs. Scatter plots analyses showed the expression signatures (Fig.1 A,C). Hierarchical clustering expression showed significant differences in the spinal cord between SCII rats and control rats (Fig.1 B,D). Among the top 10 DElncRNAs, NONRATT007222.2 and NONRATT019127.2 were the most significantly up-regulated and down-regulated in the spinal cord after SCII (Table 3). Moreover, Csf3 and Ankrd9 were the most significantly up- and down-regulated mRNAs in spinal cord after SCII (Table 4). Further, transcripts were proved to be distributed on all chromosomes (Fig. 2).

\section{Validation of IncRNAs and mRNAs}

Eight mRNAs and 8 lncRNAs were randomly selected to verify the high throughput RNA sequencing results in six sample pairs by qRT-PCR. We found that the expressions of lncRNAs NONRATT007222.2, NONRATT013040.2, TCONS_00018593 and TCONS_00008647 were up-regulated, while NONRATT019127.2, NONRATT013069.2, TCONS_00018687 and TCONS_00020362 were down-regulated. Of the eight selected mRNAs, NM_017104.2, NM_001191577.1, XM_017592198.1 and NM_013037.1 were up-regulated, and NM_001142366.2, NM_001191578.1, XM_017592225.1 and NM_001191043.1 were downregulated in the SCII model (Fig. 3). The qRT-PCR results were in line with the high throughput RNA sequencing. Therefore, the results validated our high throughput RNA sequencing profile as highly reliable and showed that the lncRNAs and mRNAs might be involved in SCII pathogenesis.

\section{Gene ontology and KEGG Pathway annotation for differential expression genes}

In GO and KEGG pathway enrichment analyses of DEmRNAs, we found 6707 mRNAs that were differentially expressed. GO enrichment analysis showed that the enriched GO biological processes for up-regulated genes in the SCII group were negative regulation of CD40 signaling pathway, positive regulation of T-helper 17 cell differentiation, cellular response to glucoside, cellular response to diterpene and cellular response to cyanide. The enriched GO cellular components for up-regulated genes in the SCII group were for the CRLF-CLCF1 complex, inner dense plaque of desmosome, outer dense plaque of desmosome, integrin alphaL-beta2 complex 
196

197

198

199

200

201

202

203

204

205

206

207

208

209

210

211

212

213

214

215

216

217

218

219

220

221

222

223

224

225

226

227

228

229

230

231

232

233

234

235

and integrin alpha1-beta1 complex. The enriched GO molecular functions for up-regulated genes in the SCII group were chemokine (C-C motif) ligand 5 binding and NEDD8 ligase activity (Fig. 4A). Down-regulated mRNAs were enriched in dendrite arborization, maintenance of synapse structure, neuron projection, axon, neuronal cell body, extracellularly glycine-gated ion channel activity, protein binding and calcium ion binding of GO biological process, cellular component and molecular function (Fig. 4B).

Similarly, differentially expressed genes were analyzed using KEGG. We found that upregulated genes in the spinal cord after SCII were involved in cytokine-cytokine receptor interaction (rno04060), TNF (rno04668) and NF-кB signaling pathway (rno04064) (Fig. 5A), while down-regulated genes were involved in axon guidance (rno04360), calcium (rno04020) and cAMP signaling pathway (rno04024) (Fig. 5B).

\section{IncRNAs/mRNAs co-expression analysis and functional prediction}

Co-expression analysis was performed to understand the roles of lncRNAs according to their coexpressed mRNAs. 385 DEmRNAs and 221 DElncRNAs, which were composed of 606 nodes and 500 edges (Figure S1) were involved. Additionally, positively co-expressed and negatively co-expressed networks were constructed to show two distinct co-expression patterns of lncRNAmRNA associated with SCII (Fig. 6A,B). Among these co-expressed mRNAs, the top three upregulated expressed DEmRNAs were Ermap, Hmox land I11r1, and these mRNAs were involved in regulation of cytokine production, apoptotic process, wound healing involved in inflammatory response, regulation of inflammatory response and positive regulation of neuron migration. The down-regulated DEmRNAs were Tmem255b, Smarcalland Cpne5. These genes are associated with many functions, such as cellular response to DNA damage stimulus, neuron projection and regulation of dendrite extension. To better predict the functions of DElncRNAs identified in our study, functional annotation of their co-expressed mRNAs were performed (Fig. 7A,B). The KEGG pathway analysis showed that the most significantly enriched pathway was the TNF signaling pathway and that, there are 62 mRNAs that might be associated with SCII among these co-expressed mRNAs.

\section{Prediction of cis-and trans-regulated genes of IncRNAs}

Cis- and trans-regulated target genes of the DElncRNAs were predicted to further explore how lncRNAs regulate the pathological process of SCII. Thirty-four lncRNAs were found to have at least five cis-regulated target genes, some of which have been reported to be associated with SCII (Fig. 8A). The top five lncRNAs ranked by the number of predicted nearby coding genes were TCONS_00026300, TCONS_00026304, TCONS_00026343, TCONS_00026342 and TCONS_00026425. LncRNAs and trans-regulated genes are displayed in Fig. 8B. lncRNAs have different numbers of potential coding genes. For example, NONRATT007903.2 had a maximum of 12 trans-regulated genes, but NONRATT028952.2 and NONRATT001746.2 only had 1 transregulated gene.

\section{Discussion}

In the present study, we identified global expression changes of lncRNAs and possible relationships with coding genes in the delayed phase of SCII for the first time. A sum of 761 up- 
236 regulated and 694 down-regulated lncRNAs were found to be significantly differentially 237 expressed 48 hours in the rat spinal cord after SCII. Accordingly, 3772 up-regulated and 2935 238 down-regulated mRNAs were identified in the SCII model, suggesting that they were likely to be 239 involved in SCII pathological processes.

240 Furthermore, we found that DElncRNAs and DEmRNAs were distributed on all chromosomes, 241 indicating multiple potential functions they might play in SCII. Eight lncRNAs and 8 mRNAs 242 were chosen for qRT-PCR, and the qRT-PCR results were in line with high throughput 243 sequencing. Among them, Aqp4 (NM_001142366.2) was reported to be associated with a protective role of HMGB1 on astrocytic swelling after oxygen-glucose deprivation(Sun et al., 2017). Study also showed that Ryr2 (NM_001191043.1) in astrocytes and axons was increased after hypoxic injury, suggesting that it might have a neuroprotective role by reducing the cellular oxidative load(Kesherwani \& Agrawal, 2012). GO and KEGG pathway enrichment analysis revealed the role of differentially regulated lncRNAs. It is well established that the NF-KB signaling pathway is closely associated with neuroinflammation and neuronal apoptosis in SCII(Sun et al., 2017; Wang et al., 2017). Induction of the cAMP signaling pathway is involved in improvement of neurologic function following SCII in mice(Mares et al., 2015). The TNF and Calcium signaling pathways are also speculated to be associated with SCII, but the precise roles of these pathways in SCII needs further research. It is not easy to predict the lncRNAs function according to their nucleotide sequences due to a lack of primary sequence conservation leading to secondary structures(Huang et al., 2011). Thus, to further reveal the biological functions of DElncRNAs in SCII, lncRNAs/mRNAs coexpression networks were constructed. In all, 385 DEmRNAs and 221 DElncRNAs were identified in the network. Based on this network we described two different co-expression patterns (positive and negative co-expression) related to SCII, which suggested distinct underlying regulation mechanisms. Further functional annotation of the DEmRNAs co-expressed with the DElncRNAs indicated that several common and meaningful pathways were enriched, including TNF, NF- $\mathrm{kB}$ and MAPK signaling pathways. The most significantly enriched pathway was the TNF signaling pathway, involving 62 co-expressed mRNAs. The TNF signaling pathway plays important roles in a variety of physiological and pathological processes, such as apoptosis, proliferation, differentiation, immune response modulation and inflammation induction(Bradley, 2008; McCoy \& Tansey, 2008). Studies have shown that ischemia reperfusion induces protein breakdown, lipid peroxidation and DNA damage, which lead to neuronal apoptosis and death, were caused by amplifying the proinflammatory response(Liu et al., 2017; Xie et al., 2017). These data suggested that the TNF signaling pathway and associated DElncRNAs might be promising targets for further investigation.

272 Finally, we predicted corresponding mRNAs of DElncRNAs for functional annotation through cis- and trans-targeting. Cis-regulation is identified as lncRNA transcription affecting expression

274 TCONS_00026304 were predicted to act on Rnf2 through cis-targeting. Nrf2 activation plays an 275 important role in spinal cord ischemic tolerance (Xu et al., 2014). Nrf2 activation was involved 
276 in the therapeutic effect of methane on SCII via mediating anti-inflammatory and anti-apoptotic

277 activities (Wang et al., 2017). In our study, the lncRNAs NONRATT026881.2,

278 TCONS_00000173 and TCONS_00032291 were predicted to act on Cdk4 in a trans fashion.

$279 \mathrm{Cdk} 4$ induction is involved in programmed cell death in SCII (Sakurai et al., 2000). Toghter, 280 most DElncRNAs in the co-expression network have not yet been annotated, it is worth 281 performing further studies to explore potential relationship between lncRNAs and SCII.

282 The major limitation of the present study is that we investigated lncRNA and mRNA changes 48 283 hours after reperfusion, but did not detect their expression profiles at different time point or 284 validate changes at the protein level. In addition, we did not select spinal cord areas for RNA 285 isolation, RNA sequencing, or RT-PCR analyses. Besides that, the number of animals included 286 in each group for RNA sequencing may be relatively small to identify DElncRNAs and 287 DEmRNAs. Research with a time course and further experiments with more animal samples are 288 needed to reveal roles of key lncRNAs and genes in SCII.

\section{Conclusions}

290 In summary, differentially expressed mRNA and lncRNA transcripts involved in SCII were 291 identified and validated using high-throughput RNA sequencing. Here, we indentified potential 292 lncRNAs involed in the pathological process of SCII by four methods: 1. DElncRNAs selected 293 with the criteria $p$-value $<0.05$ and $\mid$ fold change $\mid \geq 2 ; 2$. DElncRNAs involed in pathways 294 predicted by KEGG pathway enrichment analysis; 3. DElncRNAs co-expressed with DEmRNAs; 295 4. DElncRNAs annotated through cis- and trans-targeting with DEmRNAs. These lncRNAs 296 might be reliable candidates for further study by in vitro and vivo experiments. Our results 297 provided a basis for functional research on the lncRNAs involved in SCII. The results showed 298 that specific lncRNAs may be important for diagnosis and therapy of SCII.

\section{Acknowledgment}

300 We thank Yao Cheng for help in RNA-seq analysis. 


\section{REFERENCES}

303

304

305

306

307

308

309

310

311

312

313

314

315

316

317

318

319

320

321

322

323

324

325

326

327

328

329

330

331

332

333

334

335

336

337

338

339

340

341

342

343

344

345

346

347

348

349

350

351

352

353

354
Alkan F, Wenzel A, Palasca O, Kerpedjiev P, Rudebeck AF, Stadler PF, Hofacker IL, Gorodkin J. 2017. RIsearch2: suffix array-based large-scale prediction of RNA-RNA interactions and siRNA off-targets. Nucleic acids research 45:e60 DOI 10.1093/nar/gkw1325.

Bolger AM, Lohse M, Usadel B. 2014. Trimmomatic: a flexible trimmer for Illumina sequence data. Bioinformatics 30:21142120 DOI 10.1093/bioinformatics/btu170.

Bradley JR. 2008. TNF-mediated inflammatory disease. The Journal of pathology 214:149-160 DOI 10.1002/path.2287.

Cech TR, Steitz JA. 2014. The noncoding RNA revolution-trashing old rules to forge new ones. Cell 157:77-94 DOI 10.1016/j.cell.2014.03.008.

Fan L, Wang K, Shi Z, Die J, Wang C, Dang X. 2011. Tetramethylpyrazine protects spinal cord and reduces inflammation in a rat model of spinal cord ischemia-reperfusion injury. Journal of vascular surgery 54:192-200 DOI 10.1016/j.jvs.2010.12.030.

Hasturk A, Atalay B, Calisaneller T, Ozdemir O, Oruckaptan H, Altinors N. 2009. Analysis of serum pro-inflammatory cytokine levels after rat spinal cord ischemia/reperfusion injury and correlation with tissue damage. Turkish neurosurgery 19:353-359.

Huang R, Jaritz M, Guenzl P, Vlatkovic I, Sommer A, Tamir IM, Marks H, Klampfl T, Kralovics R, Stunnenberg HG, Barlow DP, Pauler FM. 2011. An RNA-Seq strategy to detect the complete coding and non-coding transcriptome including full-length imprinted macro ncRNAs. PloS one 6:e27288 DOI 10.1371/journal.pone.0027288.

Kesherwani V, Agrawal SK. 2012. Upregulation of RyR2 in hypoxic/reperfusion injury. Journal of neurotrauma 29:1255-1265 DOI 10.1089/neu.2011.1780.

Kim D, Langmead B, Salzberg SL, 0000000288597432 AO. 2015. HISAT: a fast spliced aligner with low memory requirements. Nature methods 12:357-360 DOI 10.1038/nmeth.3317.

Langmead B, Salzberg SL. 2012. Fast gapped-read alignment with Bowtie 2. Nature methods 9:357-359 DOI 10.1038/nmeth.1923.

Li XQ, Lv HW, Tan WF, Fang B, Wang H, Ma H. 2014. Role of the TLR4 pathway in blood-spinal cord barrier dysfunction during the bimodal stage after ischemia/reperfusion injury in rats. Journal of neuroinflammation 11:62 DOI 10.1186/1742-2094-11-62.

Liu Q, Zhang X, Dai L, Hu X, Zhu J, Li L, Zhou C, Ao Y. 2014. Long noncoding RNA related to cartilage injury promotes chondrocyte extracellular matrix degradation in osteoarthritis. Arthritis \& rheumatology 66:969-978 DOI 10.1002/art.38309.

Liu SG, Wang YM, Zhang YJ, He XJ, Ma T, Song W, Zhang YM. 2017. ZL006 protects spinal cord neurons against ischemia-induced oxidative stress through AMPK-PGC-1alpha-Sirt3 pathway. Neurochemistry international 108:230-237 DOI 10.1016/j.neuint.2017.04.005.

Liu Y, Pan L, Jiang A, Yin M. 2018. Hydrogen sulfide upregulated lncRNA CasC7 to reduce neuronal cell apoptosis in spinal cord ischemia-reperfusion injury rat. Biomedicine \& pharmacotherapy = Biomédecine \& pharmacothérapie 98:856-862 DOI 10.1016/j.biopha.2017.12.079.

Liu Z, Xie D, Zhang H, AUID- Oho. 2018. Long noncoding RNA neuroblastoma-associated transcript 1 gene inhibits malignant cellular phenotypes of bladder cancer through miR-21/SOCS6 axis. Cell death \& disease 9:1042 DOI 10.1038/s41419-018-1090-z.

Mares JM, Foley LS, Bell MT, Bennett DT, Freeman KA, Meng X, Weyant MJ, Cleveland JC Jr, Fullerton DA, Puskas F, Reece TB. 2015. Erythropoietin activates the phosporylated cAMP [adenosine 3'5' cyclic monophosphate] response element-binding protein pathway and attenuates delayed paraplegia after ischemia-reperfusion injury. The Journal of thoracic and cardiovascular surgery 149:920-924 DOI 10.1016/j.jtcvs.2014.11.011.

McCoy MK, Tansey MG. 2008. TNF signaling inhibition in the CNS: implications for normal brain function and neurodegenerative disease. Journal of neuroinflammation 5:45 DOI 10.1186/1742-2094-5-45.

Mercer TR, Dinger ME, Mattick JS. 2009. Long non-coding RNAs: insights into functions. Nature reviews. Genetics 10:155159 DOI 10.1038/nrg2521.

Ni B, Cao Z, Liu Y. 2013. Glycyrrhizin protects spinal cord and reduces inflammation in spinal cord ischemia-reperfusion injury. The International journal of neuroscience 123:745-751 DOI 10.3109/00207454.2013.796551.

Qiao Y, Peng C, Li J, Wu D, Wang X. 2018. LncRNA MALAT1 is Neuroprotective in a Rat Model of Spinal Cord IschemiaReperfusion Injury Through miR-204 Regulation. Current neurovascular research 15:211-219 DOI $10.2174 / 1567202615666180712153150$. 
Roberts A, Pachter L. 2013. Streaming fragment assignment for real-time analysis of sequencing experiments. Nature methods 10:71-73 DOI 10.1038/nmeth.2251.

Sakurai M, Hayashi T, Abe K, Itoyama Y, Tabayashi K, Rosenblum WI. 2000. Cyclin D1 and Cdk4 protein induction in motor neurons after transient spinal cord ischemia in rabbits. Stroke 31:200-207.

Smith PD, Puskas F, Meng X, Lee JH, Cleveland JC, Weyant MJ, Fullerton DA, Reece TB. 2012. The evolution of chemokine release supports a bimodal mechanism of spinal cord ischemia and reperfusion injury. Circulation 126:S110117 DOI 10.1161/CIRCULATIONAHA.111.080275.

Sun L, AUID- Oho, Li M, Ma X, Feng H, Song J, Lv C, He Y. 2017. Inhibition of HMGB1 reduces rat spinal cord astrocytic swelling and AQP4 expression after oxygen-glucose deprivation and reoxygenation via TLR4 and NF-kappaB signaling in an IL-6-dependent manner. Journal of neuroinflammation 14:231 DOI 10.1186/s12974-017-1008-1.

Wan IY, Angelini GD, Bryan AJ, Ryder I, Underwood MJ. 2001. Prevention of spinal cord ischaemia during descending thoracic and thoracoabdominal aortic surgery. European journal of cardio-thoracic surgery : official journal of the European Association for Cardio-thoracic Surgery 19:203-213.

Wang L, Yao Y, He R, Meng Y, Li N, Zhang D, Xu J, Chen O, Cui J, Bian J, Zhang Y, Chen G, Deng X. 2017. Methane ameliorates spinal cord ischemia-reperfusion injury in rats: Antioxidant, anti-inflammatory and anti-apoptotic activity mediated by Nrf2 activation. Free radical biology \& medicine 103:69-86 DOI 10.1016/j.freeradbiomed.2016.12.014.

Wang Q, Li ZX, Li YJ, He ZG, Chen YL, Feng MH, Li SY, Wu DZ, Xiang HB. 2019. Identification of lncRNA and mRNA expression profiles in rat spinal cords at various time-points following cardiac ischemia/reperfusion. International journal of molecular medicine 43:2361-2375 DOI 10.3892/ijmm.2019.4151.

Wang S, Chen X, Wang M, Yao D, Chen T, Yan Q, Lu W. 2018. Long Non-Coding RNA CYP4B1-PS1-001 Inhibits Proliferation and Fibrosis in Diabetic Nephropathy by Interacting with Nucleolin. Cellular physiology and biochemistry : international journal of experimental cellular physiology, biochemistry, and pharmacology 49:2174-2187 DOI $10.1159 / 000493821$.

Wang Y, Yang X, Sun X, Rong L, Kang M, Wu P, Ji X, Lin R, Huang J, Xue Y, Fang Y. 2018. Bone marrow infiltrated Lnc-INSR induced suppressive immune microenvironment in pediatric acute lymphoblastic leukemia. Cell death \& disease 9:1043 DOI 10.1038/s41419-018-1078-8.

Wu H, Zhao ZA, Liu J, Hao K, Yu Y, Han X, Li J, Wang Y, Lei W, Dong N, Shen Z, Hu S. 2018. Long noncoding RNA Meg3 regulates cardiomyocyte apoptosis in myocardial infarction. Gene therapy 25:511-523 DOI 10.1038/s41434-0180045-4.

Xie L, 0000-0001-5435-7767 AO, Yu S, Yang K, Li C, 0000-0001-9324-4685 AO, Liang Y, 0000-0002-1927-4875 A0. 2017. Hydrogen Sulfide Inhibits Autophagic Neuronal Cell Death by Reducing Oxidative Stress in Spinal Cord Ischemia Reperfusion Injury. Oxidative medicine and cellular longevity 2017:8640284 DOI 10.1155/2017/8640284.

Xu J, Huang G, Zhang K, Sun J, Xu T, Li R, Tao H, Xu W. 2014. Nrf2 activation in astrocytes contributes to spinal cord ischemic tolerance induced by hyperbaric oxygen preconditioning. Journal of neurotrauma 31:1343-1353 DOI 10.1089/neu.2013.3222.

Zheng R, Du M, Wang X, Xu W, Liang J, Wang W, Lv Q, Qin C, Chu H, Wang M, Yuan L, Qian J, Zhang Z. 2018. Exosome-transmitted long non-coding RNA PTENP1 suppresses bladder cancer progression. Molecular cancer 17:143 DOI 10.1186/s12943-018-0880-3.

Zhu P, Li JX, Fujino M, Zhuang J, Li XK. 2013. Development and treatments of inflammatory cells and cytokines in spinal cord ischemia-reperfusion injury. Mediators of inflammation 2013:701970 DOI 10.1155/2013/701970.

Zhu P, Zhao MY, Li XH, Fu Q, Zhou ZF, Huang CF, Zhang XS, Huang HL, Tan Y, Li JX, Li JN, Huang S, Ashraf M, Lu C, Chen JM, Zhuang J, Guo HM. 2015. Effect of low temperatures on BAX and BCL2 proteins in rats with spinal cord ischemia reperfusion injury. Genetics and molecular research : GMR 14:10490-10499 DOI 10.4238/2015.September.8.10. 
Table $\mathbf{1}$ (on next page)

qRT-PCR primer sequences 
1 Table 1 qRT-PCR primer sequences.

\begin{tabular}{lll}
\hline & Forward primer(5'->3') & Reverse primer (5'->3') \\
\hline TCONS_00018593 & TAAGGCACTCTGGGGACGAA & GGGATTAGATGCTGTGGGGC \\
TCONS_00018687 & GGACATCCCAAAGATGGCGT & GGCAGGAGCAGGTTCTTAGT \\
NONRATT013040.2 & GGCGCCTGTGAGTAGATGAA & CCGACAAAATTCGGCTCGTG \\
NONRATT013069.2 & ATCCACCGACAGTTGGAACC & GTACGCAGAATGACCTCGCT \\
NONRATT007222.2 & AGTCAAGTTCAAGCCGTCCC & CCACTAGGAGTGACCTGTGC \\
TCONS_00008647 & AGAGTCAAGAGCGGACCCA & GTCCCAAACGTGTTTGTCCT \\
NONRATT019127.2 & CTCCGTTCCGGGCTCTAGT & AACGGGGAGACAGGATGCTT \\
TCONS_00020362 & GAAAGCTGGCCTGGGTTCTA & GCTCAGGACCCTAACCTGTG \\
NM_001191578.1 & CCATGGTGACTGAGAGTCCG & CTGTCTCCAGGGACAAGCAG \\
XM_017592225.1 & GAGGCTGGAGGAAGGAGAGA & ATGTTGACGTCTGGGGTGTC \\
XM_017592198.1 & CCATGGTGACTGAGAGTCCG & CTGTCTCCAGGGACAAGCAG \\
NM_001191577.1 & CTGTGAAAAGCTGGCAACCC & TCAGGCTTCCAAACGGAGTC \\
NM_017104.2 & GTCGACAACTTTGCCACCAC & GGTACCACCACAGAATGGGG \\
NM_013037.1 & GTCAGTGCACAAAAGGCTGG & ATTTCCGCTTGGGGGCATAA \\
NM_001142366.2 & TAGCCGGTCTCCTAGCAGTT & CTTTGCCTTGCAGGTTGGTC \\
NM_001191043.1 & CACTACACTGTGAGGCCCAG & GGCTGCCTCACACTAACCAT \\
\hline
\end{tabular}

2 
Table 2 (on next page)

The results of RNA-Sequencing and clean reads mapping to the reference genome for each group 
1 Table 2 The results of RNA-Sequencing and clean reads mapping to the reference genome for each

2 group.

\begin{tabular}{ccccc}
\hline Sample & raw_reads & clean_reads & Total reads & Total mapped reads \\
\hline SCII1 & $98.19 \mathrm{M}$ & $95.49 \mathrm{M}$ & 95487232 & $93482612(97.90 \%)$ \\
SCII2 & $99.73 \mathrm{M}$ & $96.94 \mathrm{M}$ & 96938560 & $95121138(98.13 \%)$ \\
SCII3 & $99.55 \mathrm{M}$ & $96.77 \mathrm{M}$ & 96773454 & $94651018(97.81 \%)$ \\
sham1 & $99.03 \mathrm{M}$ & $95.94 \mathrm{M}$ & 95938648 & $94021084(98.00 \%)$ \\
sham2 & $98.18 \mathrm{M}$ & $95.22 \mathrm{M}$ & 95220464 & $93256257(97.94 \%)$ \\
sham3 & $99.20 \mathrm{M}$ & $96.43 \mathrm{M}$ & 96426788 & $94543525(98.05 \%)$ \\
\hline
\end{tabular}

3 
Table 3 (on next page)

Top 10 up-regulated and down-regulated IncRNAs in SCII 
1 Table 3 Top 10 up-regulated and down-regulated IncRNAs in SCII.

\begin{tabular}{|c|c|c|c|c|}
\hline ID & Locus & Length & $\log 2 F C$ & P_value \\
\hline \multicolumn{5}{|l|}{ Up-regulation } \\
\hline NONRATT007222.2 & $\operatorname{chr12:2213723:2217002:+}$ & 861 & 9.8391916 & $1.37 \mathrm{E}-07$ \\
\hline TCONS_00008647 & Chr13:70748288:70749443:+ & 597 & 8.6841803 & $2.20 \mathrm{E}-20$ \\
\hline NONRATT009530.2 & chr14:84501194:84502293:+ & 1099 & 8.6388028 & $1.12 \mathrm{E}-11$ \\
\hline NONRATT027814.2 & chr8:71937230:71941941:+ & 784 & 8.5135019 & 0.03536145 \\
\hline NONRATT004368.2 & chr10:47983254:47984472:+ & 1218 & 8.485743 & $2.71 \mathrm{E}-05$ \\
\hline NONRATT015075.2 & chr2:56892628:56893363:+ & 735 & 8.4529202 & 2.97E-08 \\
\hline NONRATT001432.2 & chr1:220835102:220836600:+ & 1498 & 8.2443526 & $1.07 \mathrm{E}-07$ \\
\hline NONRATT025081.2 & chr6:92136234:92136940:- & 706 & 8.1274884 & 0.00038525 \\
\hline NONRATT024498.2 & chr6:108087671:108090182:- & 2511 & 7.6101354 & 0.0012159 \\
\hline TCONS_00026900 & Chr7:122665947:122710909:+ & 376 & 7.5871507 & 0.02229607 \\
\hline \multicolumn{5}{|l|}{ Down-regulation } \\
\hline NONRATT019127.2 & chr3:8678461:8690286:- & 411 & -8.408993 & 7.61E-18 \\
\hline NONRATT006212.2 & chr10:97733700:97735485:- & 1785 & -8.300526 & 0.0040744 \\
\hline NONRATT026560.2 & chr7:14222096:14228950:- & 3792 & -7.64609 & 0.0021739 \\
\hline NONRATT005960.2 & chr10:85655223:85658023:- & 2800 & -6.878412 & $4.03 \mathrm{E}-12$ \\
\hline NONRATT027045.2 & chr7:117180655:117187427:- & 959 & -6.779899 & 0.00030453 \\
\hline NONRATT007881.2 & chr12:24543744:24548614:- & 1353 & -6.66036 & 0.04019041 \\
\hline NONRATT015918.2 & chr2:1791288:1791544:- & 256 & -6.650513 & 0.00339351 \\
\hline NONRATT030119.2 & chr9:73614969:73615827:+ & 280 & -6.580291 & 0.00026021 \\
\hline NONRATT004679.2 & chr10:71858906:71860354:+ & 440 & -6.467266 & 0.01227824 \\
\hline NONRATT023755.2 & chr5:156811469:156812092:- & 623 & -6.430205 & 0.00166601 \\
\hline
\end{tabular}

2 
Table 4 (on next page)

Top 10 up-regulated and down-regulated mRNAs in SCII 
1 Table 4 Top 10 up-regulated and down-regulated mRNAs in SCII.

\begin{tabular}{|c|c|c|c|}
\hline ID & Gene & $\log 2 F C$ & P_value \\
\hline \multicolumn{4}{|l|}{ Up-regulation } \\
\hline NM_017104.2 & Csf3 & 11.93599648 & $5.20 \mathrm{E}-39$ \\
\hline NM_013037.1 & Il1rl1 & 11.39596538 & 0.001080239 \\
\hline XM_006247931.3 & Slc16a3 & 11.28927512 & 0.000375611 \\
\hline NM_203493.3 & Dmp1 & 10.68101399 & 0.02825727 \\
\hline XM_008766983.2 & Il1r2 & 10.65398384 & $6.52 \mathrm{E}-06$ \\
\hline NM_012589.2 & Il6 & 10.64190268 & $3.75 \mathrm{E}-58$ \\
\hline NM_053647.1 & $\mathrm{Cxcl} 2$ & 10.34386563 & $8.60 \mathrm{E}-39$ \\
\hline XM_006246949.3 & LOC497963 & 10.14662547 & $9.86 \mathrm{E}-07$ \\
\hline NM_053822.2 & S100a8 & 9.93922593 & 0.000520228 \\
\hline NM_138522.1 & $\mathrm{Cxcl3}$ & 9.859847615 & 8.34E-20 \\
\hline \multicolumn{4}{|l|}{ Down-regulation } \\
\hline NM_001012112.1 & Ankrd9 & -7.681552228 & 0.003776415 \\
\hline XM_017600715.1 & LOC 102553010 & -7.508339301 & $1.06 \mathrm{E}-13$ \\
\hline NM_012705.1 & $\mathrm{Cd} 4$ & -7.205888701 & 0.02515971 \\
\hline XM_017599444.1 & Aasdh & -7.136997274 & 0.000145736 \\
\hline NM_001271135.1 & Ulk3 & -6.983610521 & 0.002151312 \\
\hline XM_017592918.1 & Crot & -6.929796154 & 0.000487074 \\
\hline XM_017602365.1 & Zcchc16 & -6.837049544 & 0.000795313 \\
\hline XM_008760819.2 & Cdh18 & -6.687559573 & 0.000603272 \\
\hline XM_017594898.1 & Slc4a8 & -6.631820122 & 0.009401042 \\
\hline XM_017596528.1 & Speg & -6.425796396 & 0.001340429 \\
\hline
\end{tabular}

2 


\section{Figure 1}

Expression Profiles of LncRNAs and mRNAs in Rat Spinal Cord after IschemiaReperfusion Injury

Scatter-plot for comparing global expression profiles of IncRNAs (A) and mRNAs (C) in the spinal cord between the SCII and sham-operated rats. $X$ and $Y$ axes are the mean normalized signal values (log2 scaled). Red points represent up-regulated IncRNAs or mRNAs while blue points represent down-regulated IncRNAs or mRNAs. Heat map showing hierarchical clustering of DEIncRNAs (B) and DEmRNAs (D) in the spinal cord between the SCII and shamoperated rats. The color scale indicates the expression of DEIncRNAs and DEmRNAs. Red and blue indicates up- and down-regulation, respectively. 
A

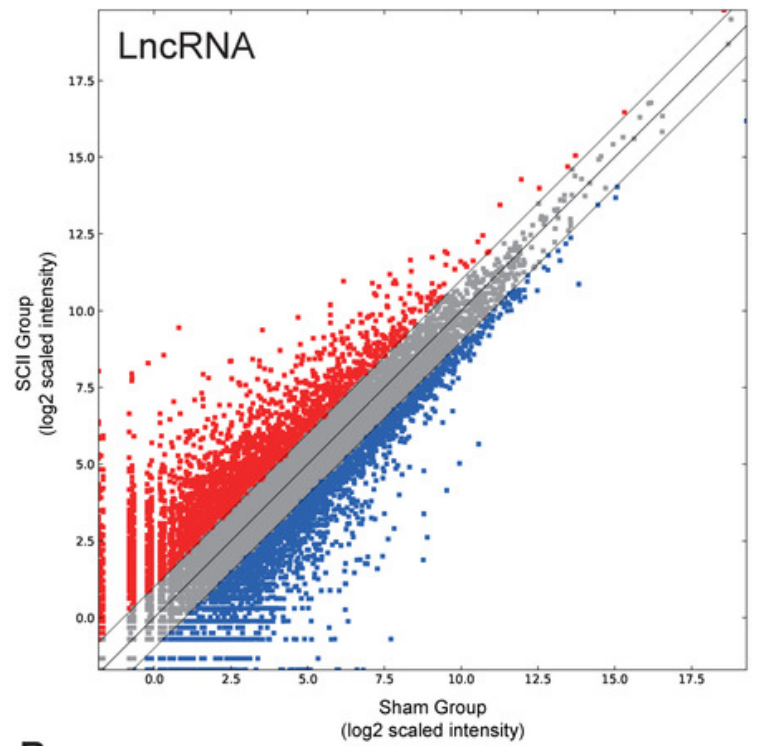

B

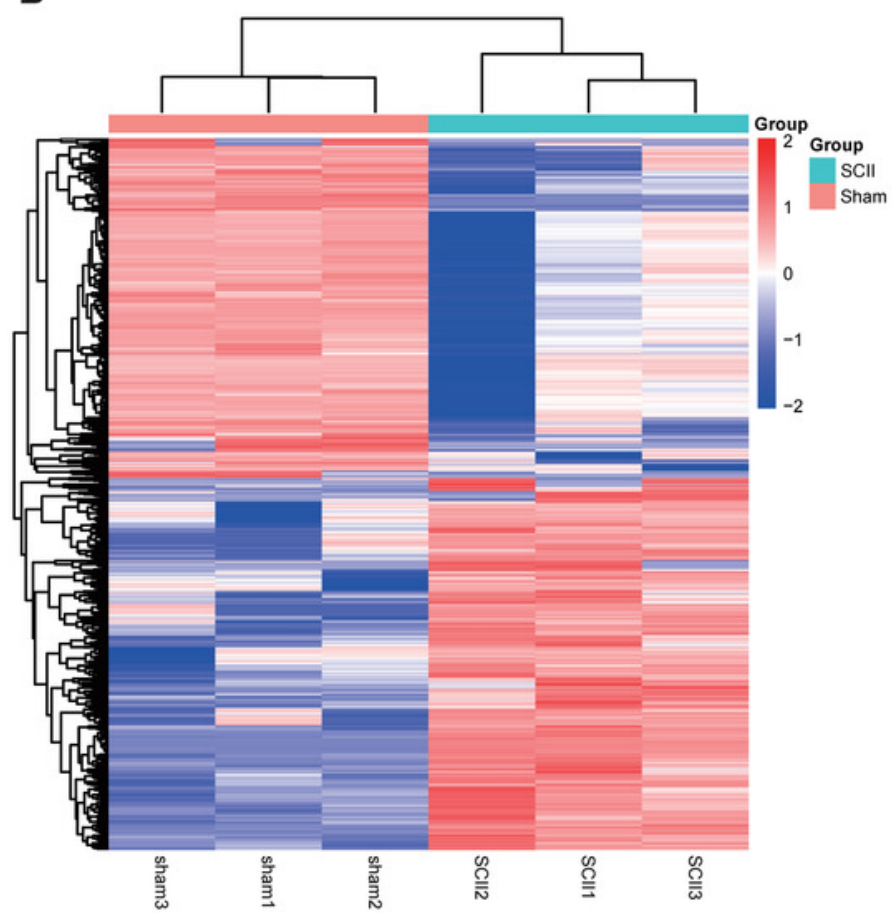

C

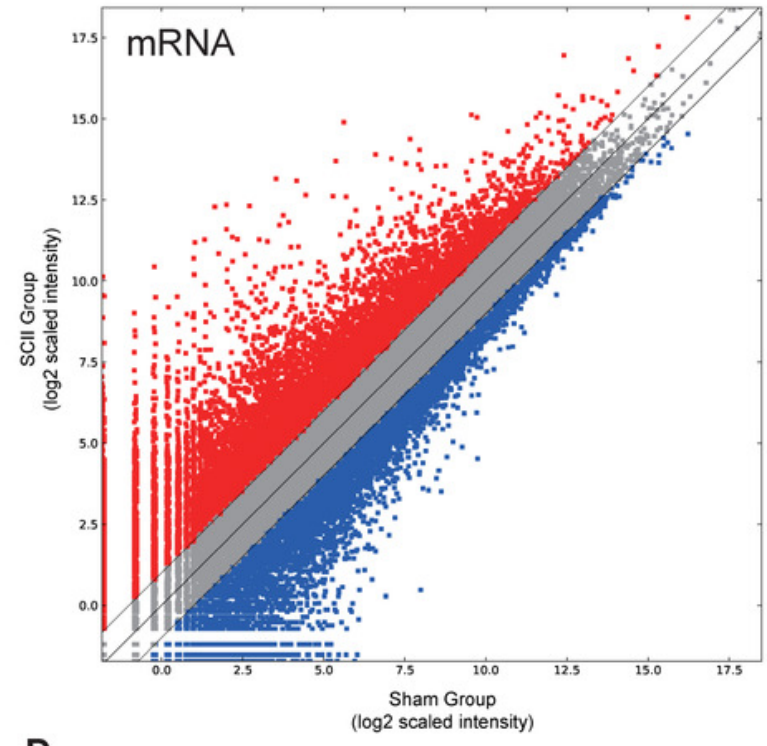

D

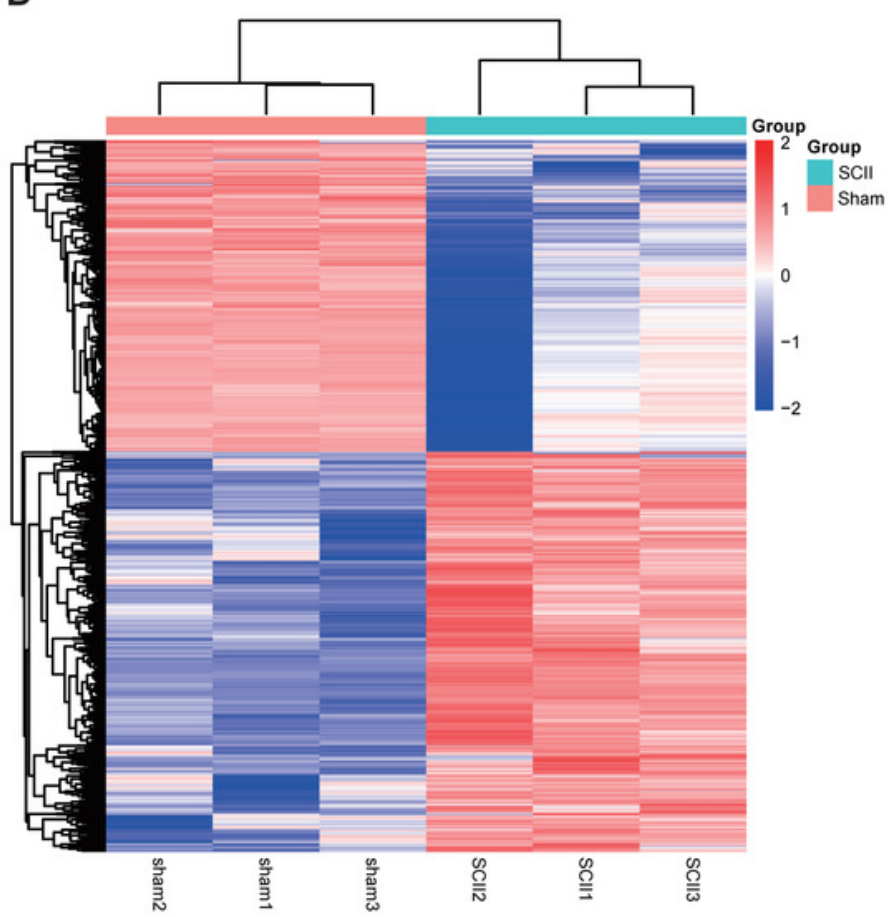




\section{Figure 2}

Circos Plots Representing the Distribution of DElncRNAs and DEmRNAs on Rat Chromosomes

The outermost layer of the circos plot is the chromosome map of the rat genome. The largest and larger inner circles represent all DElncRNAs detected by RNA-sequencing with fold change $\geq 2.0, p<0.05$, and FDR $<0.05$. The increased or decreased IncRNAs are marked with red or green bars, respectively, and bar heights in the larger inner circle indicate numbers of DEIncRNAs. The smaller and smallest inner circles represent all DEmRNAs detected by RNA-sequencing with fold change $\geq 2.0, p<0.05$ and FDR $<0.05$. Increased or decreased mRNAs are marked with red or green bars, respectively, and bar heights in the smallest inner circle indicate numbers of DEmRNAs. 


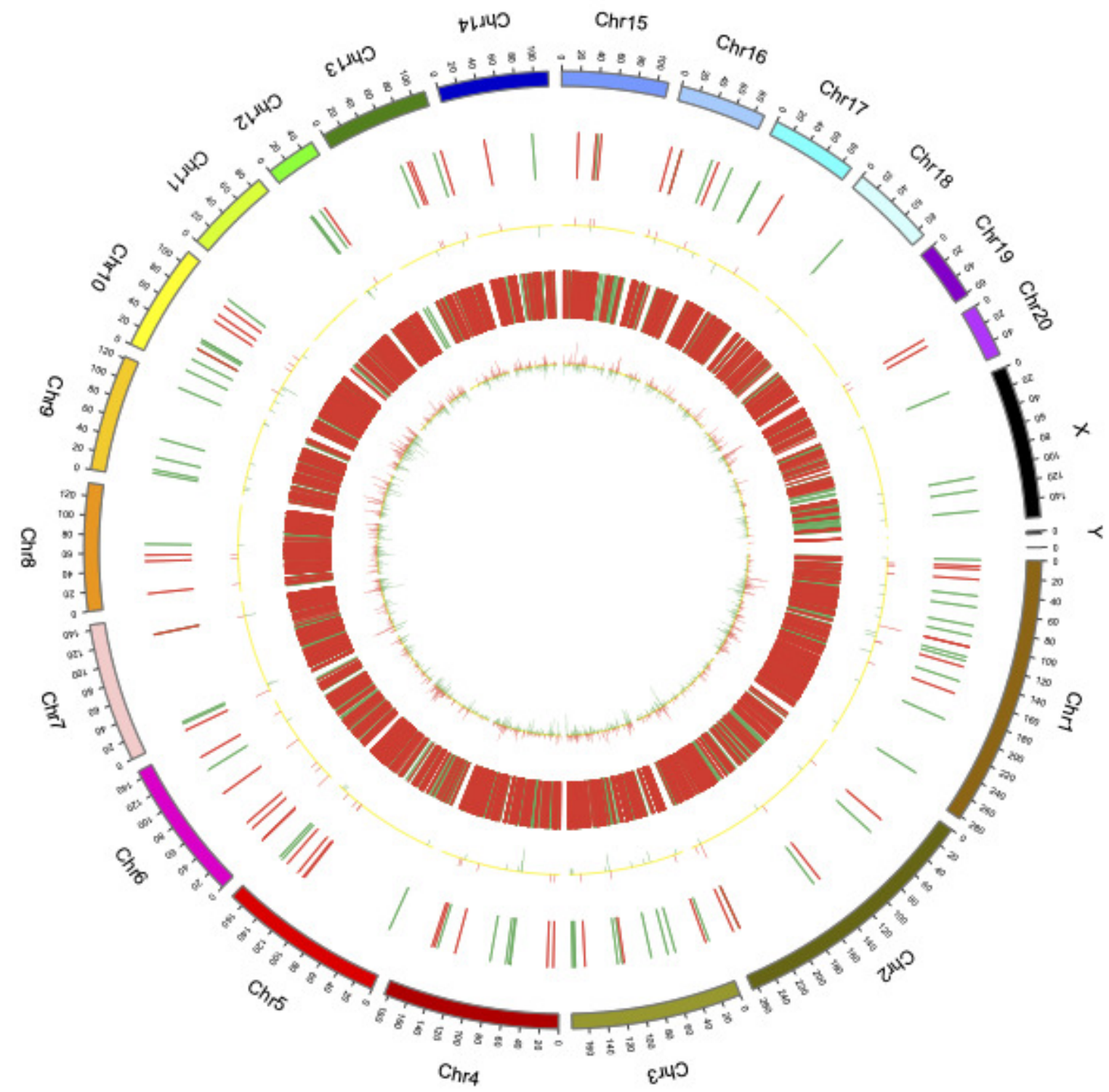


Figure 3

qRT-PCR Validation of DEIncRNAs and DEmRNAs in the Spinal Cord of SCII Rats Compared with Matched Tissues of Sham-operated Rats

(A) Expression level of DEIncRNAs determined by qRT-PCR (normalized to U6); (B) Expression level of DEmRNAs determined by qRT-PCR (normalized to GAPDH). 
A

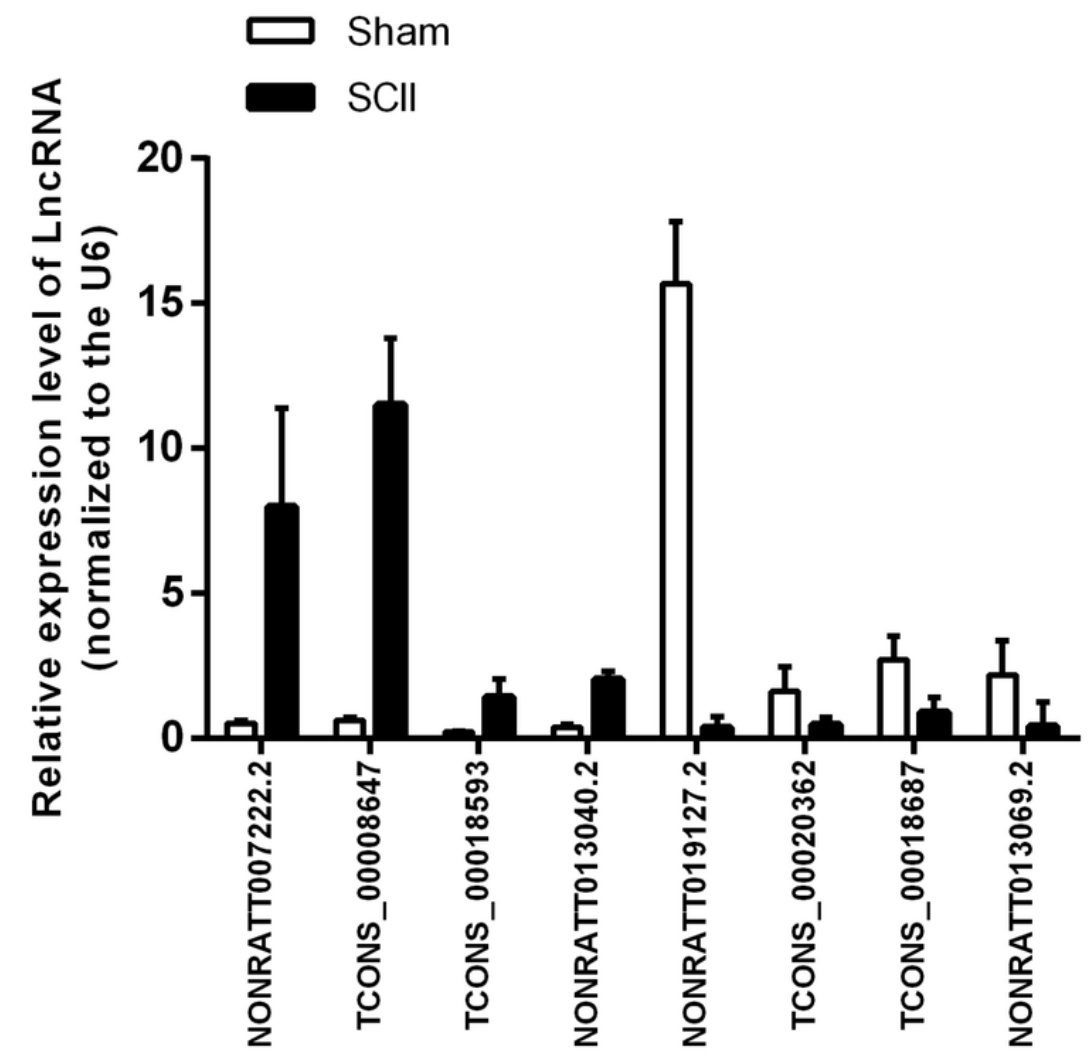

B

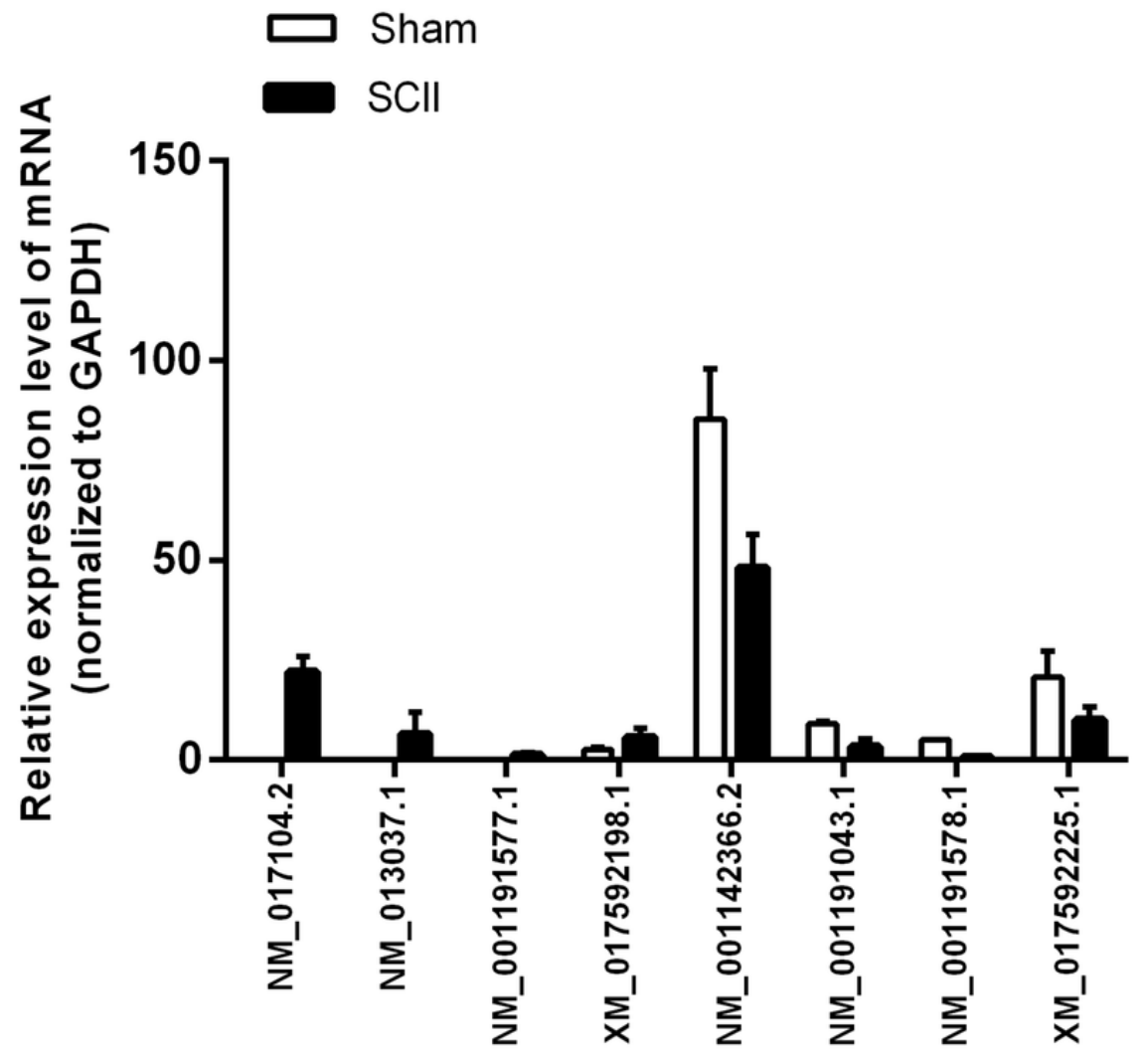




\section{Figure 4}

GO Enrichment Analysis for the DEmRNAs with the 10 Highest Enrichment Scores

(A) GO enrichment analysis for up-regulated DEmRNAs; (B) GO enrichment analysis for downregulated DEmRNAs; red bars are biological processes, green bars are cellular components, and blue bars are molecular functions. The ordinate is the -Log10 P-value (-LgP). Larger - LgP values correlate with smaller p-values, indicating that the enrichment of differentially expressed genes in a given pathway is significant. 

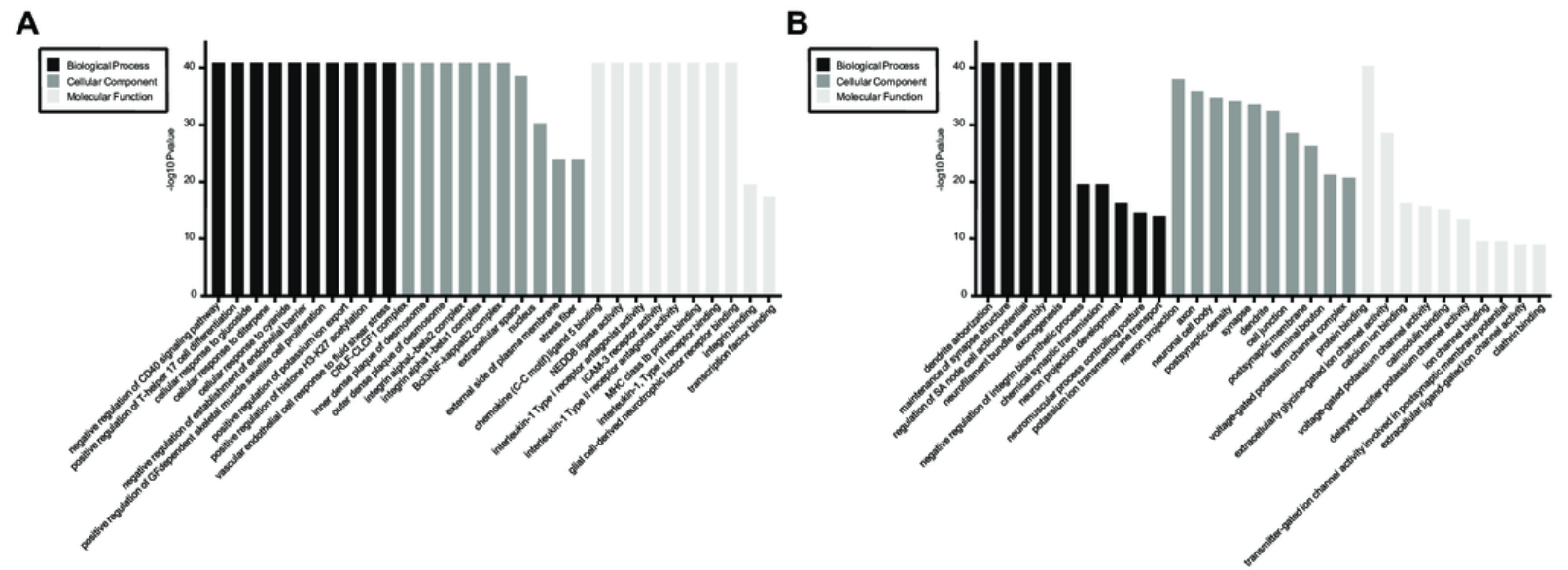


\section{Figure 5}

KEGG Pathway Enrichment Analysis of the DEmRNAs with the 20 Highest Enrichment Scores

(A) KEGG pathway enrichment analysis for up-regulated DEmRNAs; (B) KEGG pathway enrichment analysis for down-regulated DEmRNAs. The abscissa is the enrichment score. Size represents the number of enriched genes, and color indicates the degree of enrichment. Higher enrichment scores correlate with lower P-values, indicating that the enrichment of differentially expressed genes in a given pathway is significant. 

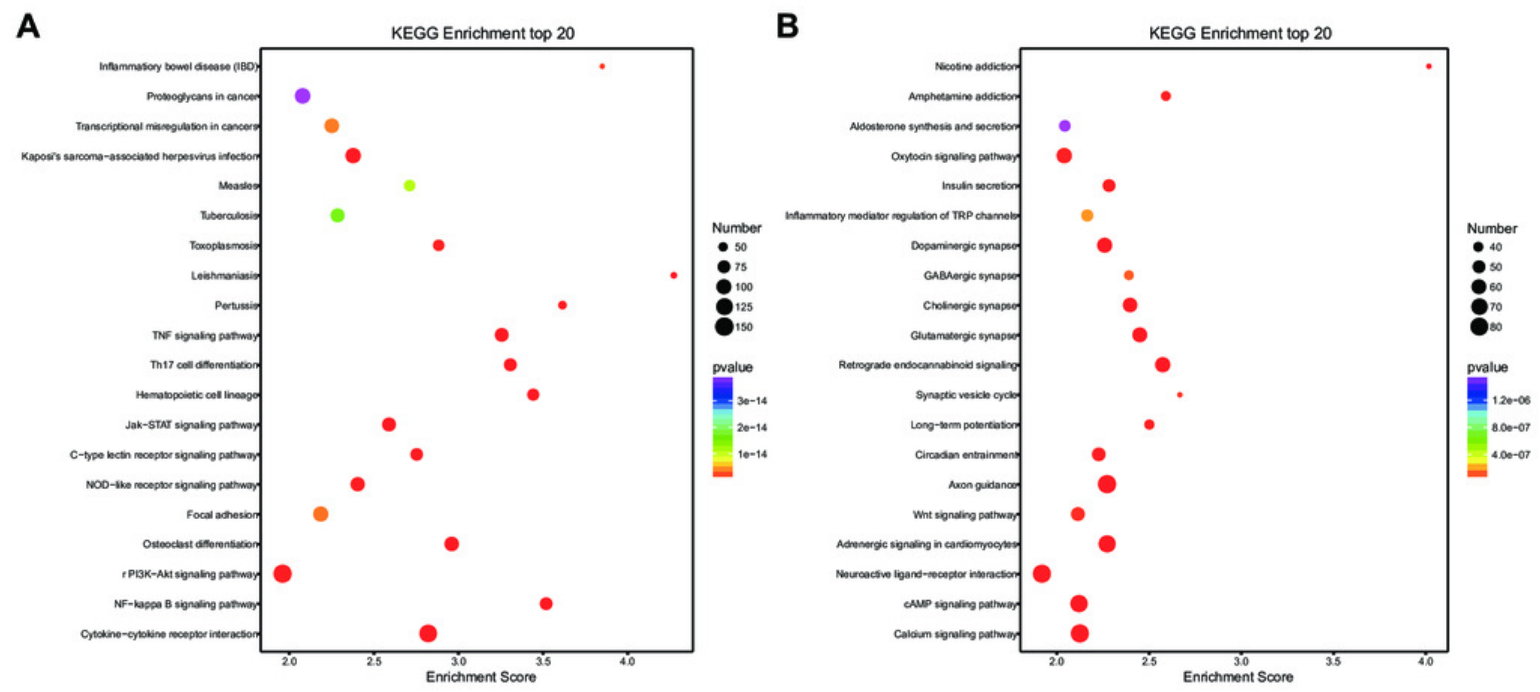


\section{Figure 6}

Co-expression Network of the DEIncRNAs and DEmRNAs

(A) Positive co-expression network and (B) negative co-expression network of DElncRNAs and DEmRNAs. Arrows represent DEIncRNAs, and ellipse nodes represent DEmRNAs. Red lines indicate positive co-expression relationships while blue lines indicate negative co-expression relationships between DEIncRNAs and DEmRNAs. 

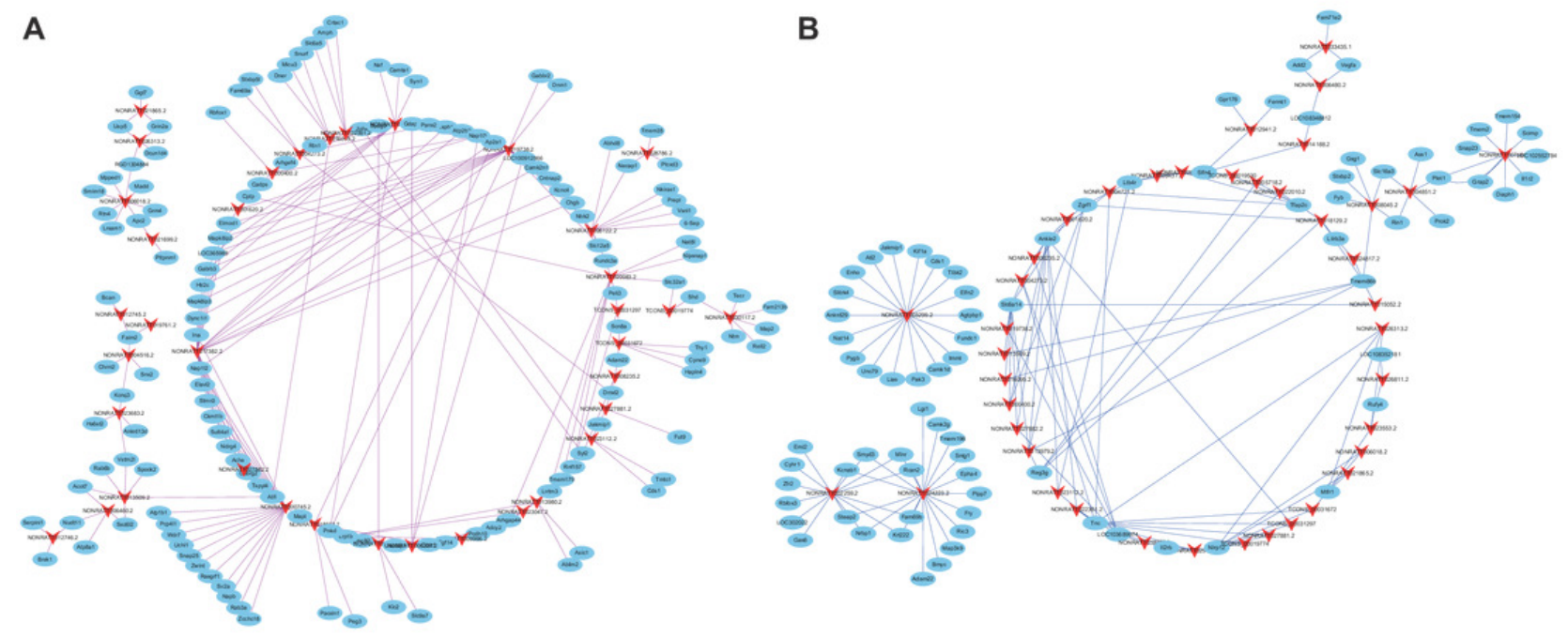


\section{Figure 7}

\section{Functional Annotation for Co-expressed DEmRNAs of DEIncRNAs}

(A) GO enrichment analysis for co-expressed DEmRNAs of DEIncRNAs. Red bars are biological processes, green bars are cellular components, and blue bars are molecular functions. (B) KEGG pathway enrichment analysis for co-expressed DEmRNAs of DEIncRNAs. The size of the spot indicates the gene numbers enriched in the pathway, and the color of the spot indicates the significance level of the enriched pathway. 


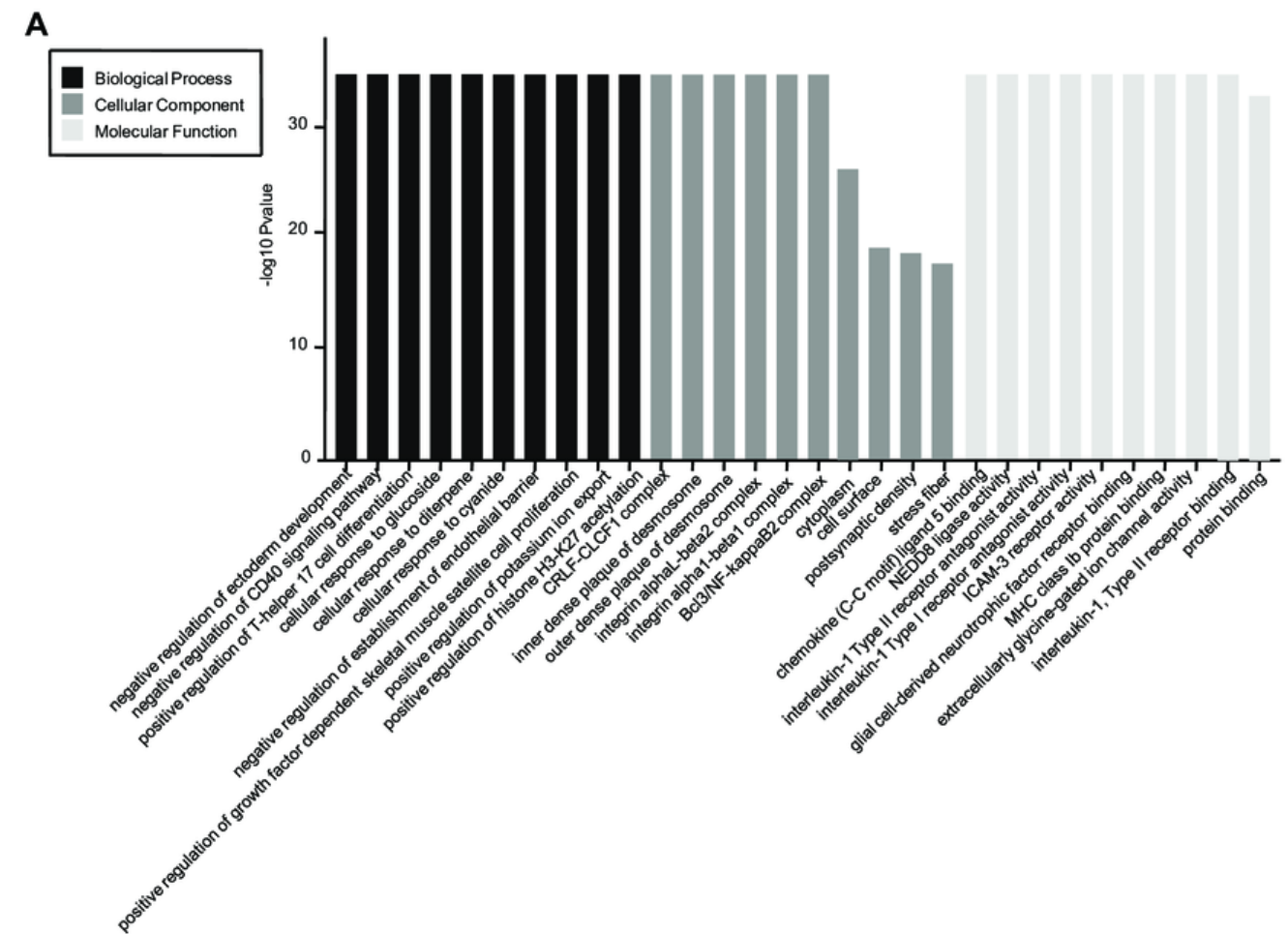

B

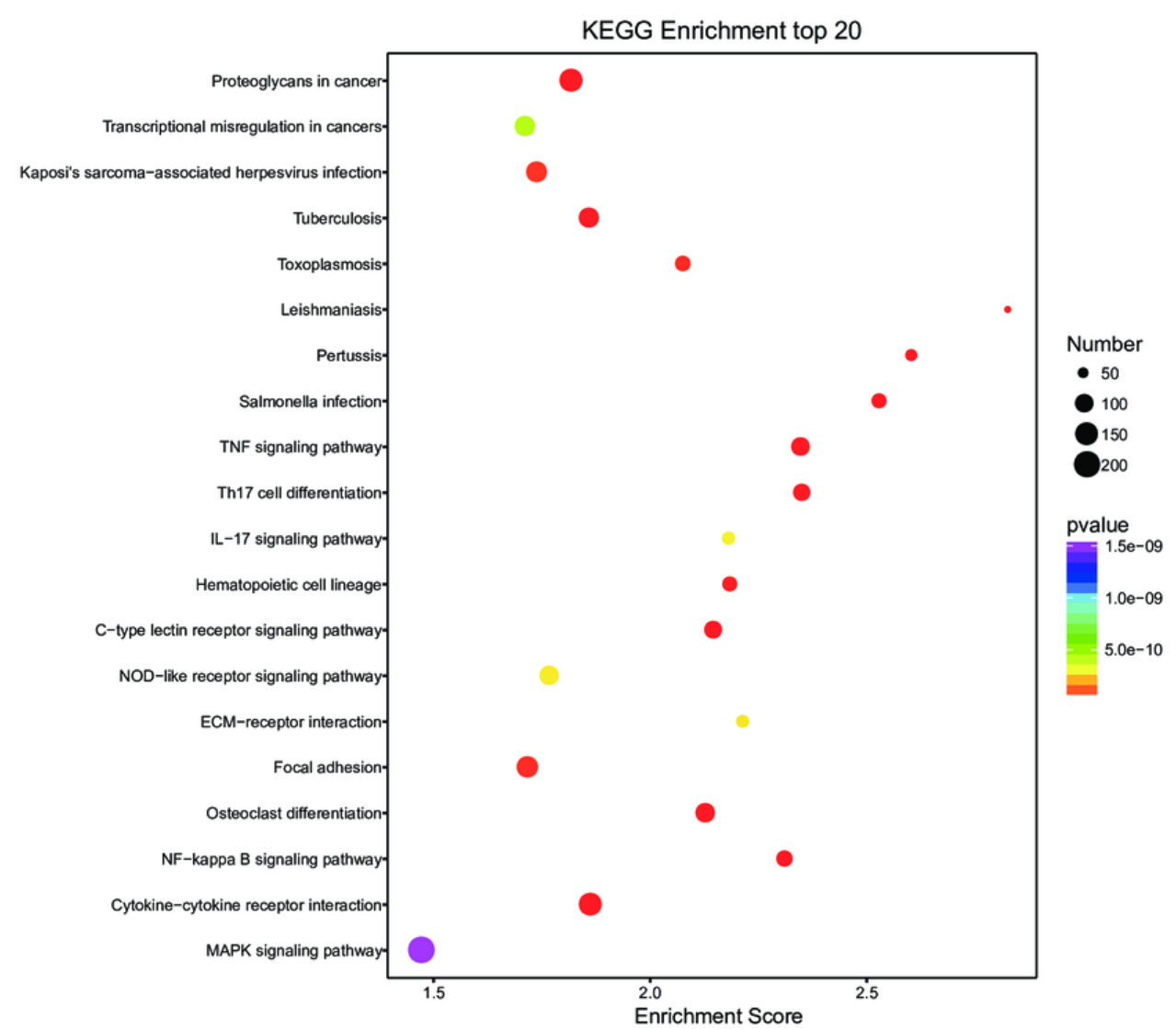


Figure 8

Construction Networks of the DEIncRNAs and Their Cis- and Trans-regulated DEmRNAs

(A) IncRNAs and their potential trans-regulated genes; (B) IncRNAs and their potential cisregulated nearby genes. Arrows represent DEIncRNAs, while the ellipse nodes represent trans- or cis-regulated DEmRNAs. Nrf2 and Cdk4 are marked in yellow. 
A

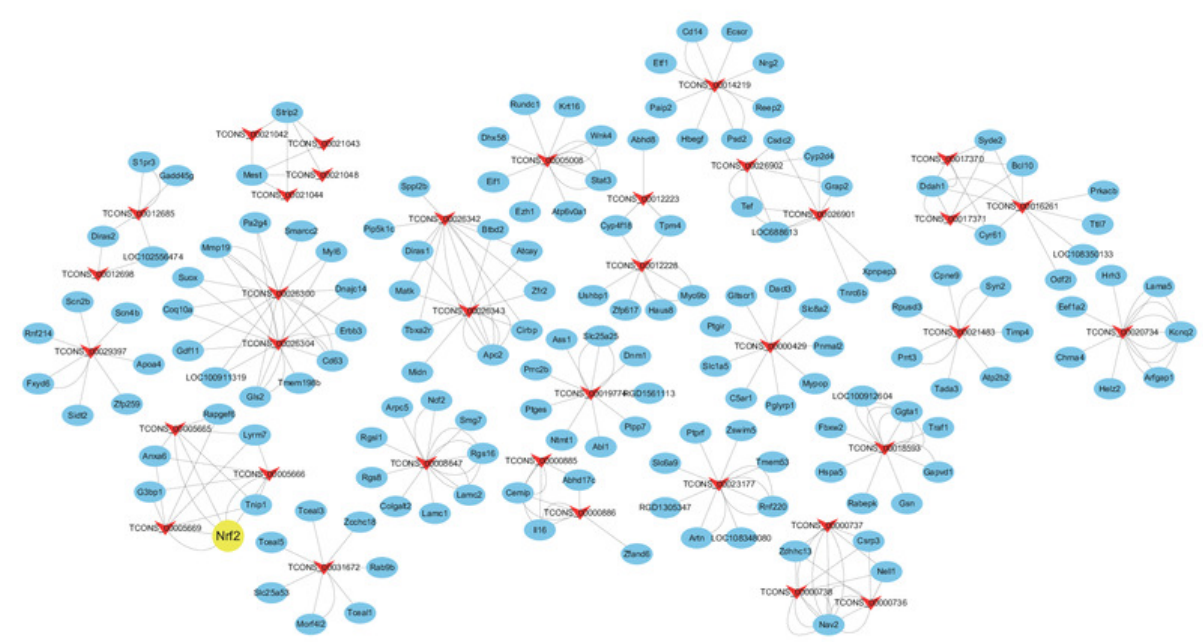

B

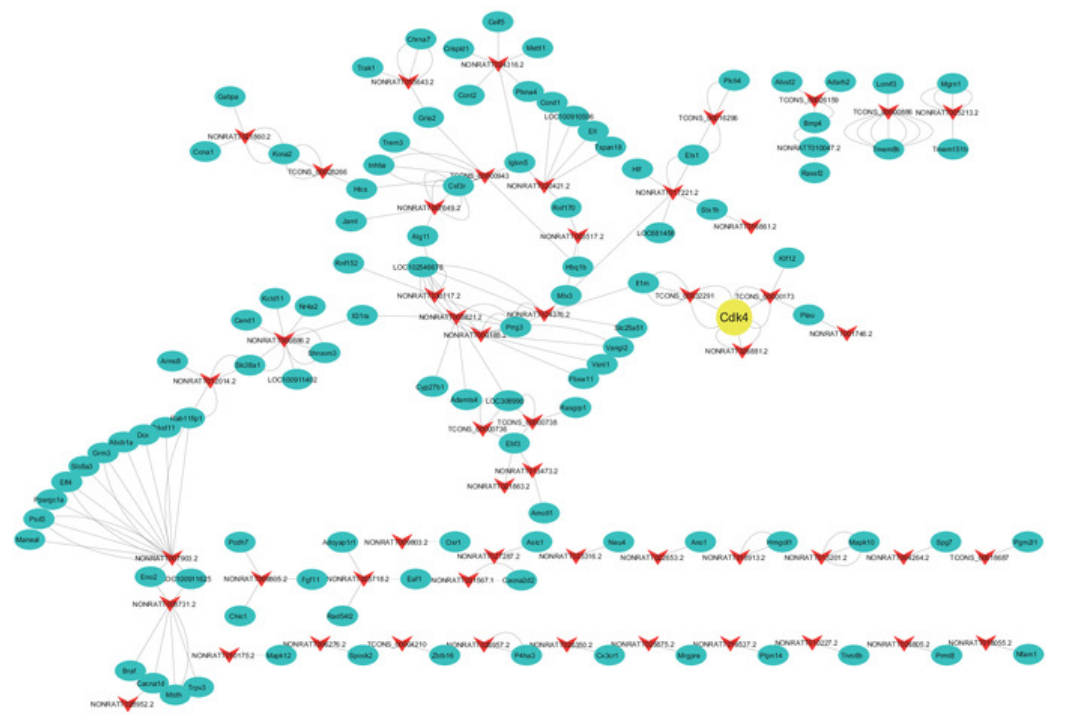

